\title{
BMJ Open Quality Meta-analysis of interventions to reduce door to needle times in acute ischaemic stroke patients
}

\author{
Michael Siarkowski (D) , ${ }^{1}$ Katie Lin, ${ }^{2}$ Shari S Li, ${ }^{3}$ Abdulaziz Al Sultan, ${ }^{4}$ \\ Heather Ganshorn (D) , ${ }^{5}$ Noreen Kamal, ${ }^{6}$ Michael Hill, ${ }^{4}$ Eddy Lang ${ }^{2}$
}

To cite: Siarkowski M, Lin K, Li SS, et al. Metaanalysis of interventions to reduce door to needle times in acute ischaemic stroke patients. BMJ Open Quality 2020;9:e000915. doi:10.1136/ bmjoq-2020-000915

- Additional material is published online only. To view please visit the journal online (http://dx.doi.org/10.1136/ bmjoq-2020-000915)

2016 Critical Care Conference (Toronto, ON Canada).

Received 12 January 2020

Revised 24 June 2020

Accepted 9 July 2020

D Check for updates

(C) Author(s) (or their employer(s)) 2020. Re-use permitted under CC BY-NC. No commercial re-use. See rights and permissions. Published by BMJ.

${ }^{1}$ Dentistry, The University of British Columbia, Vancouver, British Columbia, Canada

${ }^{2}$ Cumming School of Medicine, University of Calgary, Calgary, Alberta, Canada

${ }^{3}$ Medicine, Faculty of Health Sciences, Queen's University, Kingston, Ontario, Canada ${ }^{4}$ Department of Clinical Neurosciences, University of Calgary, Calgary, Alberta, Canada

${ }^{5}$ Sciences, University of Calgary, Calgary, Alberta, Canada

${ }^{6}$ Faculty of Engineering, Dalhousie University, Halifax, Nova Scotia, Canada

Correspondence to Dr Eddy Lang;

Eddy.Lang@albertahealthser vices.ca

\section{ABSTRACT}

Background Door-to-needle time (DTN) has an important impact on thrombolysis and reperfusion outcomes in the treatment of acute ischaemic stroke. This systematic review is a critical synthesis of studies evaluating DTN reduction strategies.

Method Ovid MEDLINE, PubMed, Cochrane Database of Systematic Reviews, CINAHL, ProQuest dissertations and LILACS were used as bibliographic databases for primary literature. CIHI, Health Quality Council of Alberta, Health Quality Ontario and websites of heart and stroke associations in Canada, USA, UK, Australia and New Zealand were used as sources of grey literature. Searched reports were screened by title and abstract, and full texts were located for review. Articles quality was evaluated using National Institute of Health's Study Quality Assessment tools. Methods for improving DTN were categorised under 13 DTN reduction strategies, primarily adapted from the Target: Stroke Phase II recommendations, and including two additional categories: Strategies not encompassed by any Target: Stroke recommendation, and Combinations of Interventions. Results 96 studies (4 randomised control trials, 1 review, 91 observational pre/post studies) were included in the review. All strategies and interventions resulted in a reduction of DTN. Approaches using combinations of interventions were the most effective at reducing DTN (33.77\% DTN reduction, standard mean difference $=1.857$, $95 \% \mathrm{Cl}=1.510-2.205)$, and were more effective than approaches using only a single strategy $(p=0.040)$. DTN reduction was associated with the duration of the DTN reduction programme at each facility $(p=0.006)$.

Interpretation The greatest reductions in DTN were observed when implementing combinations of DTN reduction strategies, although there was no significant advantage to implementing more than two strategies simultaneously.

PROSPERO registration number 42016036215.

\section{INTRODUCTION}

Thrombolysis has been shown to improve stroke outcomes by reducing disability, ${ }^{1}$ and it is most effective when administered early. ${ }^{23}$ Performance measurement of an acute stroke service can be evaluated based on the time from patient arrival at the hospital to the time of alteplase (tissue plasminogen activator (tPA) ) administration or door-to-needle time (DTN). ${ }^{4}$ From stroke onset to alteplase administration, multiple teams (Emergency Medical System (EMS), care centre emergency department, acute stroke team) work in coordination to deliver care. Healthcare providers possess varying knowledge, skills and resources to care for acute stroke patients, and thus the logistics of delivering effective and efficient care can be challenging. However, it is possible to consistently reduce the DTN through targeted procedural and system-level changes. ${ }^{45}$ The American Stroke Association implemented the Target: Stroke programme in 2010 to provide guidelines for achieving DTN of less than 60 min. ${ }^{46}$ The programme was updated in 2015 with Target: Stroke Phase II, which built on the success of the first phase and provided organisational recommendations for improving care facility DTN. ${ }^{7}$ Despite the Target: Stroke campaign's success, there is currently no published systematic evaluation of which recommended strategies are most effective for improving systems of acute stroke care. The lack of guidance is particularly noticeable when contrasted with other time-sensitive DTN initiatives affecting patient prognosis and outcomes, such as those studied in acute coronary syndrome systems of care.

To address the knowledge gap, we performed a systematic review and metaanalysis of all published DTN reduction initiatives to date. The goal of the systematic review is to clearly identify the most effective strategies for improving and reducing DTN to provide a recommendation for acute ischaemic stroke care improvement. The broader learnings from the review may be useful beyond the scope of intravenous alteplase treatments, with applicability to door-to-puncture time and door-to-balloon time initiatives as well. This study adds to the current body of knowledge by summarising and comparing interventions to reduce DTN for acute ischaemic stroke. We hypothesise 
Table 1 Patient Intervention Comparison Outcome: PICO statement

\section{PICO aspect}

$\begin{array}{ll}\text { Population } & \text { Care facilities admitting patients for treatment of acute ischaemic stroke with intravenous thrombolytic therapy. } \\ \text { Intervention } & \text { The review will only consider studies where a door-to-needle optimisation programme was in place, wherein } \\ \text { the goal of the programme was to reduce the time from suspected acute ischaemic stroke patient intake at a } \\ \text { care facility until the point where they received thrombolytic therapy. }\end{array}$

that some DTN reduction strategies would result in greater DTN improvement than others. This review aimed to answer the question: for care facilities admitting patients for treatment of acute ischaemic stroke with intravenous thrombolytic therapy, which interventions to reduce in-hospital delays, when compared with other strategies, have been most effective at reducing DTN?

\section{METHODS}

\section{Protocol and registration}

The review adhered to recommendations in the Preferred Reporting Items for Systematic Reviews and MetaAnalyses (PRISMA) statement and registered with PROSPERO (accessible from the NHS PROSPERO website http://www.crd.york.ac.uk/PROSPERO/). Drafting was performed according to MOOSE guidelines. ${ }^{8}$

\section{Patient involvement}

This research was done without patient involvement. Patients were not invited to comment on the study design and were not consulted to develop patient relevant outcomes or interpret the results. Patients were not invited to contribute to the writing or editing of this document for readability or accuracy.

\section{Literature search}

A qualified health sciences librarian (at least MLIS, or equivalent) conducted searches of Ovid MEDLINE, PubMed, Cochrane Database of Systematic Reviews, CINAHL, ProQuest dissertations and the LILACS database based on the Patient Intervention Comparison Outcome (PICO) statement outlined in table 1. CIHI, Health Quality Council of Alberta, Health Quality Ontario and websites of heart and stroke associations in Canada, USA, UK, Australia and New Zealand were used as sources of grey literature. Hand-searching through reference lists of obtained articles was performed on articles passing full-text screening. Databases and grey literature sources were last searched on 1 August 2019, covering all publication date ranges to 1 August 2019. Figure 1 shows our detailed search strategy, which was informally peer-reviewed by another health sciences library prior to execution.

Database(s): Ovid MEDLINE(R) Epub Ahead of Print, In-Process \& Other Non-Indexed Citations, Ovid MEDLINE(R) Daily and Ovid MEDLINE(R) 1946 to Present

\begin{tabular}{|l|l|l|}
\hline$\#$ & Searches & Results \\
\hline 1 & exp Stroke/ & 114774 \\
\hline 2 & stroke ${ }^{\star} . k f$, tw. & 214735 \\
\hline 3 & 1 or 2 & 247376 \\
\hline 4 & exp Thrombolytic Therapy/ or exp Tissue plasminogen activator/ & 34381 \\
\hline 5 & (thromboly* or tissue plasminogen activator or tpa).kf,tw. & 59271 \\
\hline 6 & 4 or 5 & 70947 \\
\hline 7 & (door-to-needle or dtn or dnt or door-to-thrombolysis or in-hospital delay ${ }^{*}$ or onset-to-treatment or interval time* or triage-to-treatment or door-to-CT or treatment time or tPA time).kf,tw. & 15341 \\
\hline 8 & 3 and 6 and 7 & 710 \\
\hline
\end{tabular}

Figure 1 Example of detailed search strategies for used databases. Search strategy included all studies available through the identified databases as of 1 August 2019. 


\section{Study selection}

We included studies assessing the efficacy of DTN reduction strategies. A DTN reduction strategy was any method implemented at a care centre with intent to reduce the time between hospital entry and administration of intravenous thrombolytic agent to acute ischaemic stroke patients. The study inclusion criteria were as follows: (1) patients must be initially diagnosed with acute ischaemic stroke, (2) study patients must receive thrombolytic treatment (eg, alteplase) via intravenous route, (3) the study must record time taken from hospital entry until administration of intravenous thrombolytic therapeutic, (4) a formal protocol, provision or plan for reducing DTN must have been implemented over the course of the study, (5) comparative data must be present, with a clear result as to margins of improvement post implementation of DTNimprovement protocol versus pre-implementation at the same facilities.

Only full records in English or translated to English were considered as no group member was sufficiently fluent in other languages to translate otherwise available studies. Records subject to change or updates (eg, abstracts) were rejected from consideration. All study designs were included. No restriction was placed on publication date.

Independently, three reviewers reviewed titles and abstracts retrieved from the search and two reviewers assessed the eligibility of the full papers corresponding to records passing the initial screen. Both screen phases were carried out by two or more reviewers independently following achievement of an acceptable inter-rater reliability score (Cohen's kappa coefficient). Disagreements on study rating were resolved through arbitration by the principal investigator.

\section{Data extraction}

Data were independently extracted from eligible reports and entered into a pre-designed spreadsheet (online supplementary table 1). Extracted study characteristics were established a priori ((1) duration of strategy implementation (months), (2) hospital type (teaching vs community), (3) registration with a database (yes/no), (4) health practitioner type (neurology vs emergency), (5) stroke centre designation, (6) the use of combination interventions) and post hoc ((7) start date before or after European Collaborative Acute Stroke Study III based on group discussion and consensus of factors that have had historical effects or those that might bear an effect).

Individual article quality and risk of bias was assessed by two reviewers and evaluated for various characteristics, including confounders, using the NIH Quality Assessment Tools (available at https://www.nhlbi.nih.gov/ health-topics/study-quality-assessment-tools), specifically 'Quality Assessment Tool for Observational Cohort and Cross-Sectional Studies', 'Quality Assessment of CaseControl Studies', 'Quality Assessment Tool for Pre-Post Studies with No Control Group' and 'Quality Assessment Tools of Controlled Intervention Studies'. Study quality ratings of good, fair and poor were from the NIH website.
Publication bias was assessed by the Tweedie \& Duval (2000) Trim and Fill test with random-effects modelling.

Evidence quality for DTN reduction categories was assessed using the GRADEpro application (available at http://gdt.guidelinedevelopment.org/app/) to better assess certainty of findings. Observational studies were given default assessments of low quality, and randomised controlled trials (RCTs) were high quality by default, with additional downgrades or upgrades pertaining to Grading of Recommendations Assessment Development and Evaluation (GRADE) criteria.

Corresponding authors were contacted with an information request for studies missing outcome data but otherwise relevant and appropriate per the selection criteria. Contact with authors followed the general form laid out in online supplementary figure 1.

\section{Statistical methods}

The principal summary measure was standard mean difference (SMD) calculated from the mean of pre-intervention and post-intervention DTN. The SMD statistic (Cohen's $d$, calculated as mean difference between pre-intervention and post-intervention DTNs, divided by SD) ${ }^{9}$ was selected to represent DTN reduction associated with each strategy. The rationale for the selection of SMD was to provide a statistic that could be compared across studies and strategies; SMD allows for accurate and absolute comparison of strategy efficacy by controlling for studies with exceptionally low or high starting DTNs where reductions are likely to be smaller or larger. ${ }^{9}$ Relative reduction (as per cent DTN reduction) was included to improve accessibility of results, however, was not used to make comparisons between categories due to limitations of inaccuracy of the statistic. Relative DTN reduction was calculated by averaging \% reduction of each study within a category. Significance of overall DTN improvement was calculated by paired t-test of preintervention and post-intervention means.

Eleven strategies for improving DTN were adapted directly from the Target: Stroke Phase II Campaign Manual (2014) recommendations to reduce DTN, with two additional categories for studies employing multiple, simultaneous DTN reduction initiatives and studies employing DTN reduction strategies outside of the Target: Stroke Phase II recommendations (figure 2). The 13 DTN reduction categories included: (1) EMS PreNotification, (2) Stroke Tools, (3) Rapid Triage Protocol and Stroke Team Notification, (4) Single Call Activation System, (5) Transfer Directly to CT/MRI Scanner, (6) Rapid Acquisition and Interpretation of Brain Imaging, (7) Rapid Lab Testing (including Point of Care), (8) Mix tPA Ahead of time, (9) Rapid Access and Administration of intravenous tPA, (10) Team-based Approach, (11) Prompt Data Feedback, (12) Strategies not encompassed by any Target: Stroke recommendation and (13) Combinations of Interventions. For all studies employing Combination Interventions, the results were pooled into a single category for meta-analysis; unique combinations were not treated as separate combinations, as the number 


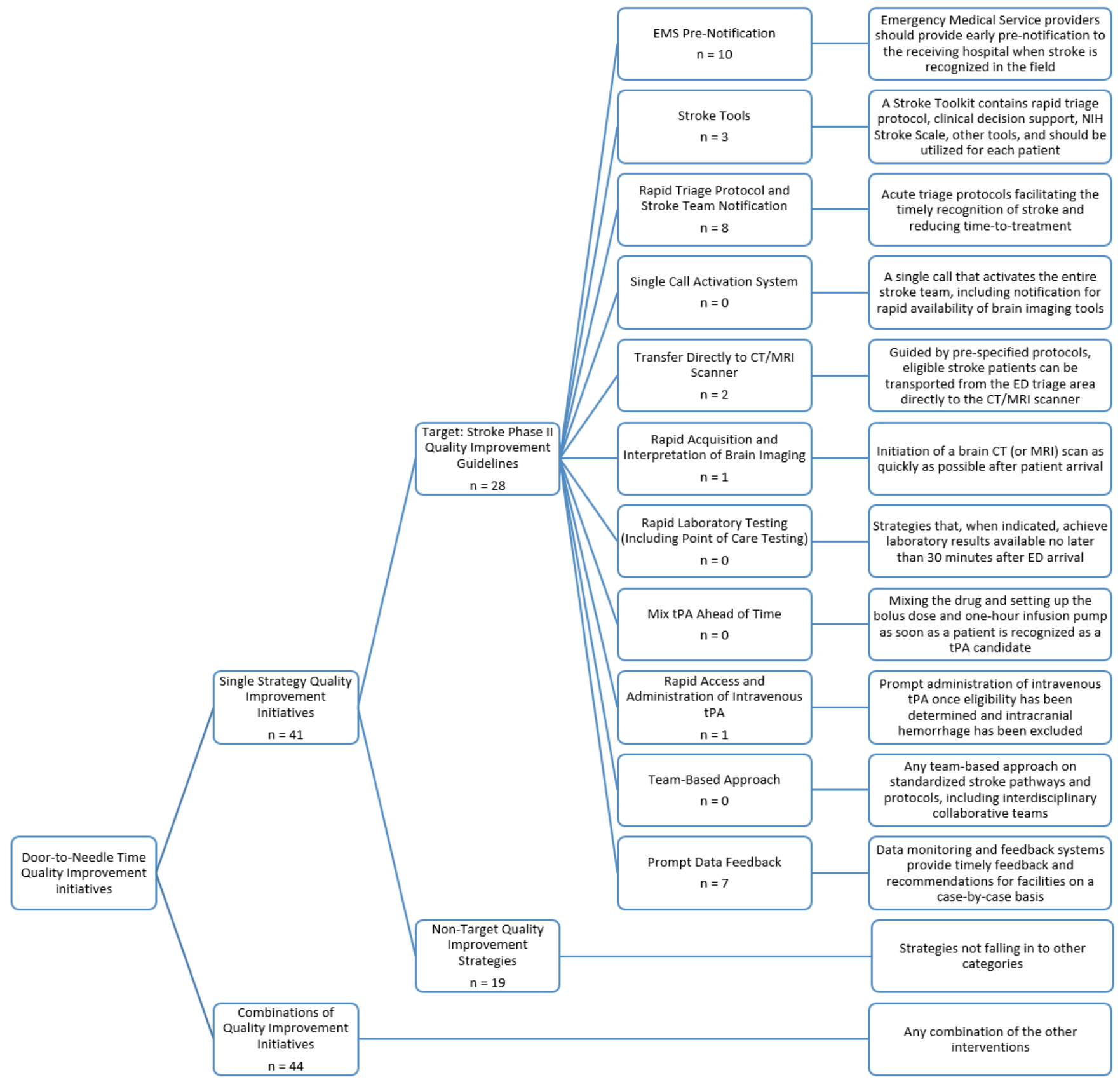

Figure 2 List of reviewed quality improvement categories. Recommendations 1-11 were adapted from Target: Stroke Phase II. Category 12 (Non-Target Quality Improvement Initiatives) was added to capture quality improvement initiatives not falling under any of the recommendations in Target: Stroke Phase II. Category 13 (Combinations of Quality Improvement Initiatives) was added to include studies employing multiple strategies simultaneously. Number of studies (n) listed under each category heading. ED, Emergency Department; NIH, National Institute of Health; tPA, tissue plasminogen activator.

of unique possibilities would have reduced the likelihood of adequate representation in the literature, preventing meta-analysis. However, a meta-regression was performed to determine if use of a strategy within a combination was more efficacious in reducing DTN than other strategies.

Analyses were conducted using STATAV.14. We conducted direct and respective conversion of medians and interquartile ranges to means and SD using the method outlined in $\$ 7.7 .3 .5$ of the Cochrane Handbook ${ }^{10}$ as needed.

Analyses were done assuming random-effects modelling to account for heterogeneity inherent in grouping similar but non-identical DTN reduction strategies. Studies in any category may encompass different implementations that abide by the original definition (eg, Rapid Laboratory Testing may present as relocation of patient tissue sampling location or relocation of labs), introducing inherent noise to the data and making a random-effects model an appropriate choice. The $\tau^{2}$ statistic was consequently chosen to represent between-study variance.

Meta-regression was performed for the seven study characteristics listed in the 'Data extraction' section. 


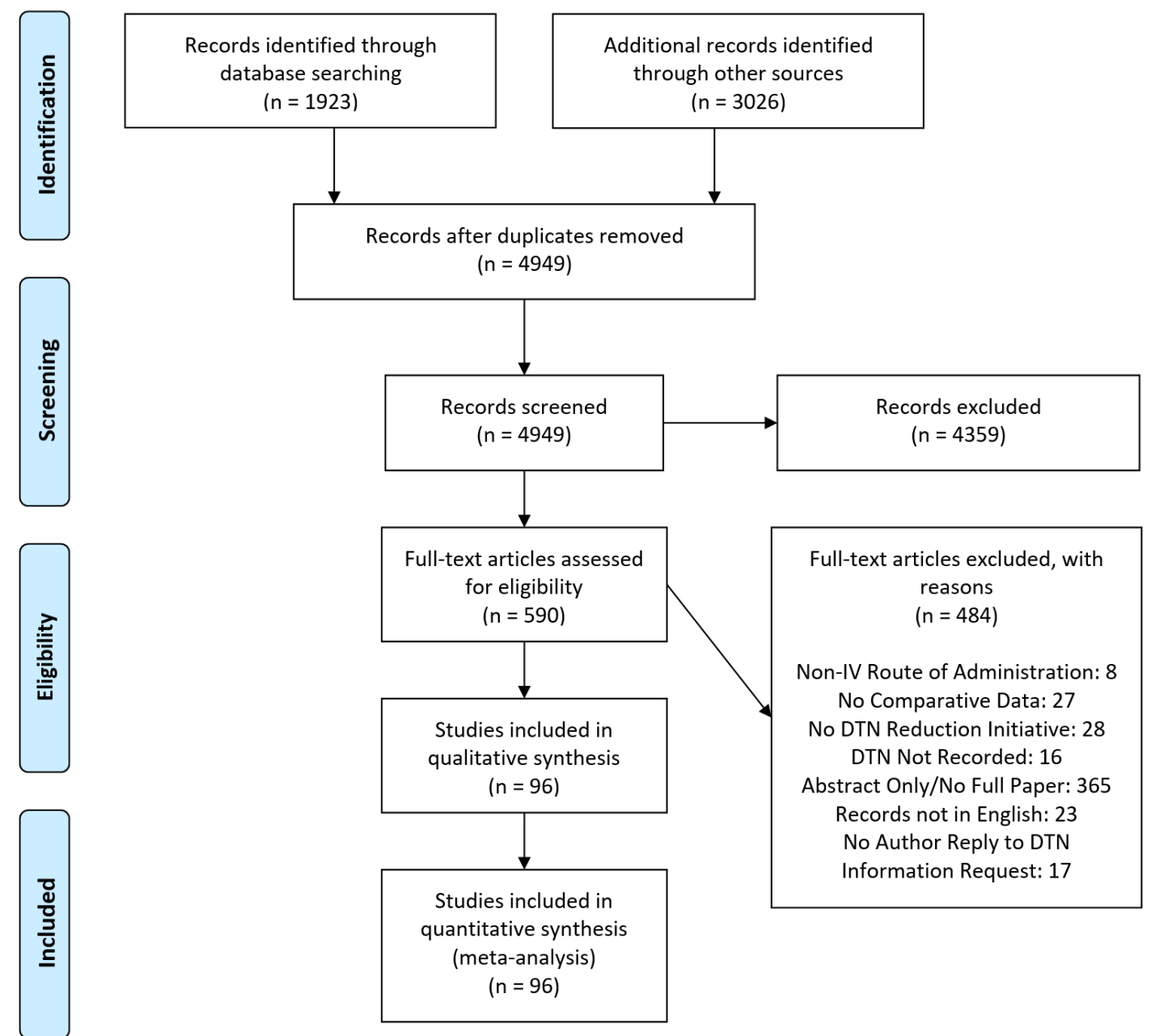

Figure 3 Summary of evidence search and selection. DTN, door-to-needle time.

Subgroup meta-analyses were performed for separate study types (observational, RCT).

Two sensitivity analyses were performed, (1) removing the three most heavily-weighted studies and (2) removing studies with 'Poor' ratings on quality assessment with the respective assessment tool.

\section{RESULTS}

\section{Search results}

Figure 3 shows the PRISMA flow chart and trial selection process. Ninety-six reports (1 review/meta-analysis, 4 RCTs, 91 pre/post observational studies) were selected for analysis. No studies examined Single-call Activation
Systems, Rapid Lab Testing, Mixing tPA Ahead of Time or Team-based Approaches as single-reduction strategies, resulting in each respective category being dropped from meta-analysis. All strategies were implemented as part of at least one Combination approach, and thus retained for within-Combination analyses.

Inter-rater reliability assessment of articles is shown in table 2 and exceeded calculated requirements.

\section{Primary analyses and study characteristics}

There were 96 studies involving 397545 acute ischaemic stroke patients. Pooled DTN improvement for all interventions was $\mathrm{SMD}=1.418\left(95 \% \mathrm{CI}=1.243-1.594, \tau^{2}=0.694\right.$,

\begin{tabular}{lllllll}
\hline Table 2 & Inter-rater agreement scores for screening \\
\hline Screening stage & Rater $\mathbf{1}$ & Rater $\mathbf{2}$ & Inter-rater agreement (\%) & Expected agreement (\%) & Cohen's kappa & SE \\
\hline Title and abstract & AAS & KL & 71.91 & 55.31 & 0.4367 & 0.0804 \\
& AAS & MS & 83.03 & 55.31 & 0.6247 & 0.1025 \\
& MS & KL & 84.27 & 55.31 & 0.6480 & 0.1060 \\
Full text & MS & NK & 92.86 & 56.38 & 0.8363 & 0.1890
\end{tabular}

Pilot phase for title and abstract screening carried out over 90 papers with each reviewer assigned to 60 papers, with overlap such that each paper was scored by two reviewers. 
$\mathrm{p}<0.001)$. Meta-analyses are presented as a Forest plot in figure 4. Record characteristics and the results of individual studies are detailed in online supplementary table 1 . Heterogeneity $\left(\tau^{2}\right)$ could not be calculated for singlestudy categories, and only exceeded a value of 1 for the Combination Strategy category.

Within the 30 studies employing a Combination approach, the number of DTN reduction strategies applied ranged from 2 to 10, however, there was no significant association between number of implemented strategies and DTN improvement $(\mathrm{p}=0.255)$. Within combination approaches, meta-regression found no significant association between each of the Target: Stroke strategies and DTN improvement, however, did find that Non-Target Strategies were associated with DTN improvement $(\mathrm{p}=0.02)$ (table 3).

\section{Meta-regression}

Duration (per month) of the DTN reduction programmes at each facility $(r=0.018, p=0.006)$ showed significant association with DTN improvement. Reductions in DTN were more strongly associated with Combination strategies vs Single-strategy approaches $(p=0.016)$. No other factor was significantly associated with DTN reduction (online supplementary table 2).

\section{Publication bias and quality}

Funnel plots were inspected for evidence of publication bias. Tweedie \& Duval (2000) Trim and Fill test using random-effects modelling imputed 51 additional studies beyond the 96 original records ( $\mathrm{p}=0.005$ ).

Study quality was reported for individual studies in online supplementary tables $3-5$. Individual study quality ranged from Poor to Good, with 16 studies ranked as low-quality (1 RCT, 10 pre/post observational) 72 studies ranked as fair-quality (1 review/meta-analysis, 2 RCT, 54 pre/post) and 8 studies ranked as being Good quality (1 RCT, 7 pre/post observational).

Overall quality of the evidence, including rationale for upgrading and downgrading, was assessed by GRADE and displayed in online supplementary table 6 .

\section{INTERPRETATION}

The pooled DTN improvement among all studies was positive, indicating a benefit to engaging in acute ischaemic stroke DTN reduction activities regardless of the chosen strategy. Combinations of DTN reduction strategies were determined to be the most effective method for reducing DTN. Within the studies using combinations of DTN reduction strategies, Prehospital Notifications or NonTarget Strategies were each individually associated with significant reductions in DTN, although there was no significant DTN reduction observed when increasing the total number of strategies implemented. Single-strategy initiatives represented the second through seventh most effective intervention types.

Based on SMD outcomes for DTN reduction (table 4), the best initiatives in descending order of effectiveness were: Combinations of DTN reduction strategies, Prompt Data Feedback, EMS Pre-Notification, Non-Target Interventions and Rapid Triage Protocol and Stroke Team Activation. A low number of studies for the Stroke Tools strategy $(n=3)$, Transfer Directly to CT/MRI strategy $(n=2)$, Rapid Acquisition and Administration of intravenous tPA strategy $(\mathrm{n}=1)$, and Rapid Acquisition and Interpretation of Brain Imaging strategy $(n=1)$ resulted in these DTN reduction strategies being dropped from a ranked comparison due to risk of bias and imprecision.

Direct-to-CT/MRI transfer was the best Single DTN reduction strategy, however, lack of weight behind the strategy may cause policymakers to fall back to the third best Single-strategy initiative, Prompt Data Feedback, which was supported by a larger number of studies $(n=7)$, maintained a high relative improvement value, and was supported by a Moderate certainty rating from the GRADE table.

From the meta-regression, the duration of the DTN reduction initiative was the only variable universally associated with reduced DTN. Heterogeneity was not above the threshold for serious consideration $\left(\tau^{2} \geq 1\right)$ for any Single-strategy approach, despite the expected diversity of studies in at least the Non-Target Intervention category. Tests for Single-strategy publication bias were deemed inappropriate due to the small $(\leq 10)$ numbers of studies in each category, as per $\$ 7.7 .3 .5$ of the Cochrane Handbook. ${ }^{10}$

\section{Limitations}

Restricting the review to English language articles excluded 23 studies from consideration. It is unclear if these studies would have been relevant on full-text review, however, it is worthwhile to consider that addition of these studies may have eliminated the pre-sensitivity analysis publication bias. If these studies were relevant to the review, it is possible that our findings could have been altered, changing the conclusions made in this research. Seventeen studies directly relevant to the review were omitted due to lack of key DTN improvement information and failure to respond to information requests. It is possible that incorporation of these missing-information studies could have affected relative ranking among the single-DTN reduction strategies. Not all Target: Stroke Phase II recommendations were individually represented in the literature. A final limitation is that the outcome measures do not represent feasibility of implementation. The implementation of some strategies may be more appropriate to specific care facilities than others, and low-cost strategies such as Prompt Data Feedback may not require exorbitant costs or restructuring to implement effectively.

\section{Conclusions}

The greatest reductions in DTN were observed in healthcare facilities implementing combinations of DTN reduction strategies, although there was no observed benefit to implementing more than two strategies simultaneously. 


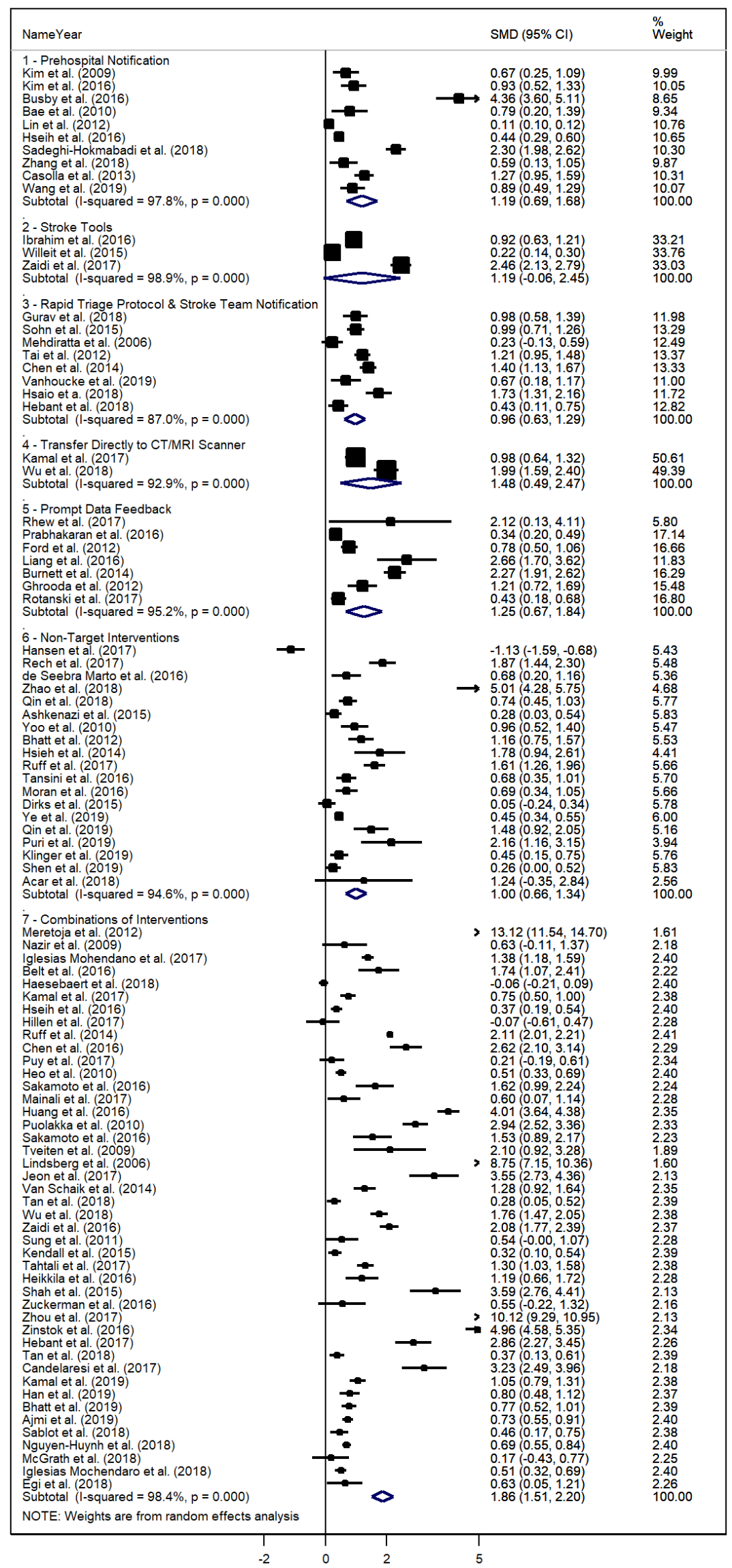

Figure 4 Forest plot of standardised mean differences (SMD) for studies, with $95 \% \mathrm{Cl}$, and weighting for individual studies and categories of quality improvement (QI) initiatives of acute ischaemic stroke management pathways by category. 
Table 3 Meta-regression of single DTN reduction strategies within Combination Interventions against standard mean difference of door-to-needle time reduction

\begin{tabular}{|c|c|c|c|c|c|}
\hline Variable & Coefficient & SE & $\mathbf{t}$ & $p>|t|$ & $95 \% \mathrm{Cl}$ \\
\hline Stroke Tools & 1.206 & 0.978 & 1.23 & 0.227 & -0.788 to 3.201 \\
\hline Single Call Activation System & -0.559 & 1.542 & -0.36 & 0.72 & -3.706 to 2.587 \\
\hline Transfer Directly to CT/MRI & -0.76 & 1.154 & -0.66 & 0.515 & -3.114 to 1.594 \\
\hline Rapid Lab Testing (including PoC) & 0.423 & 1.201 & 0.35 & 0.727 & -2.026 to 2.873 \\
\hline Mixed tPA Ahead of Time & 1.824 & 1.413 & 1.29 & 0.206 & -1.056 to 4.706 \\
\hline Rapid Access and Administration of tPA & 0.597 & 1.059 & 0.56 & 0.577 & -1.563 to 2.757 \\
\hline Team-Based Approach & -0.387 & 0.983 & -0.39 & 0.696 & -2.392 to 1.617 \\
\hline Prompt Data Feedback & 0.174 & 1.135 & 0.15 & 0.879 & -2.14 to 2.489 \\
\hline
\end{tabular}

DTN, door-to-needle time; tPA, tissue plasminogen activator.

Of the Target: Stroke Phase II DTN reduction strategies represented, Prompt Data Feedback was associated with the highest observed GRADEPro quality (moderate) and is the foremost recommended Single-strategy approach for improving DTN based on reviewed studies. DTN was inversely related with the duration of a DTN reduction initiative, suggesting some benefit to continued DTN reduction campaigns over time. Under-representation of many Target: Stroke Phase II DTN reduction strategies limits the confidence of the review's comparative findings. Future work should consider investigation into the feasibility of DTN reduction strategy implementation to better inform healthcare professionals and policy makers on appropriate DTN reduction strategies.

Acknowledgements The authors would like to thank the Alberta Health Services for providing grant funding to the research.

Contributors MS is responsible for project coordination, record screening, data extraction, statistical analysis, drafting. KL assisted with record screening, data extraction, drafting and editing for content and clarity. SSL, AAS and NK assisted with record screening, data extraction, drafting, statistical analysis and editing for content and clarity. HG developed and carried out search strategy and methodology, assisted with draft editing for content and clarity. MH oversaw meta-analysis

Table 4 Relative ranking of door-to-needle time (DTN) quality improvement strategies

\begin{tabular}{lll}
\hline $\begin{array}{l}\text { Standard mean } \\
\text { difference } \\
\text { improvement }\end{array}$ & $\begin{array}{l}\text { Standard mean difference } \\
\text { DTN improvement rank }\end{array}$ & $\begin{array}{l}\text { Relative } \\
\text { (\%) DTN } \\
\text { reduction }\end{array}$ \\
\hline 1.857 & Combination Strategies & 33.77 \\
1.255 & Prompt Data Feedback & 30.74 \\
1.185 & EMS Prehospital Notification & 26.16 \\
0.999 & Non-Target Interventions & 25.04 \\
\hline
\end{tabular}

Ranking is performed according to standard mean differences derived in the meta-analysis. Ranking descends from most improved (greatest DTN reduction) to least improved. Relative improvement calculated from mean DTN values for each study and category. execution, contributed to search strategy development, assisted with statistical analysis, assisted with draft editing for content and clarity. EL oversaw metaanalysis execution, contributed to search strategy development, assisted with draft editing for content and clarity.

Funding This project was funded by the Alberta Health Services-Emergency Strategic Clinical Network 2016 Systematic Review Grant Competition.

Competing interests MS reports grants from Alberta Health Services rewarded for the 2016 Systematic Review Grant Competition, during the conduct of the study. MH reports personal fees from Merck, non-financial support from Hoffmann-La Roche Canada Ltd, grants from Covidien (Medtronic), grants from BoehringerIngleheim, grants from Stryker Inc., grants from Medtronic LLC, grants from NoNO Inc., outside the submitted work. In addition, MH has a patent Systems and Methods for Assisting in Decision-Making and Triaging for Acute Stroke Patients issued to US Patent office Number: 62/086,077 and owns stock in Calgary Scientific Incorporated, a company that focuses on medical imaging software, is a director of the Canadian Federation of Neurological Sciences, a not-for-profit group, is a director of Circle NeuroVascular Inc. and has received grant support from Alberta Innovates Health Solutions, CIHR, Heart \& Stroke Foundation of Canada, National Institutes of Neurological Disorders and Stroke.

Patient and public involvement Patients and/or the public were not involved in the design, or conduct, or reporting, or dissemination plans of this research.

Patient consent for publication Not required.

Provenance and peer review Not commissioned; externally peer reviewed.

Data availability statement Data available upon request.

Open access This is an open access article distributed in accordance with the Creative Commons Attribution Non Commercial (CC BY-NC 4.0) license, which permits others to distribute, remix, adapt, build upon this work non-commercially, and license their derivative works on different terms, provided the original work is properly cited, appropriate credit is given, any changes made indicated, and the use is non-commercial. See: http://creativecommons.org/licenses/by-nc/4.0/.

ORCID iDs

Michael Siarkowski http://orcid.org/0000-0002-6469-377X

Heather Ganshorn http://orcid.org/0000-0002-0410-4232

\section{REFERENCES}

1 Hacke W, Kaste M, Fieschi C, et al. Intravenous thrombolysis with recombinant tissue plasminogen activator for acute hemispheric stroke. The European cooperative acute stroke study (ECASS). JAMA 1995:274:1017-25.

2 Hacke W, Donnan G, Fieschi C, et al. Association of outcome with early stroke treatment: pooled analysis of ATLANTIS, ECASS, and NINDS rt-PA stroke trials. Lancet 2004;363:768-74. 
3 Saver JL, Fonarow GC, Smith EE, et al. Time to treatment with intravenous tissue plasminogen activator and outcome from acute ischemic stroke. JAMA 2013;309:2480-8.

4 Fonarow GC, Smith EE, Saver JL, et al. Improving door-to-needle times in acute ischemic stroke: the design and rationale for the American heart Association/American stroke association's target: stroke initiative. Stroke 2011:42:2983-9.

5 Vlahaki D, Fiaani M, Milne WK. A door-to-needle time of 30 minutes or less for myocardial infarction thrombolysis is possible in rural emergency departments. CJEM 2008;10:429-33.

6 American Stroke Association. Target: stroke campaign manual. Dallas: American Stroke Association, 2011. http://www. strokeassociation.org/idc/groups/heart-public/@wcm/@hcm/@gwtg/ documents/downloadable/ucm_308277.pdf
7 American Stroke Association. Target: stroke phase II campaign manual. Dallas: American Stroke Association, 2014. http://www. strokeassociation.org/idc/groups/heart-public/@wcm/@gwtg/ documents/downloadable/ucm_470730.pdf

8 Stroup DF, Berlin JA, Morton SC, et al. Meta-analysis of observational studies in epidemiology: a proposal for reporting. Meta-analysis of observational studies in epidemiology (MOOSE) group. JAMA 2000;283:2008-12.

9 Cohen J. Statistical power analysis for the behavioral sciences. 2nd edn. Hillsdale, NJ: Erlbaum, 1988.

10 Higgins JPT, Thomas J, Chandler J, et al, eds. Cochrane Handbook for Systematic Reviews of Interventions version 6.0 (updated July 2019). Cochrane, 2019. www.training.cochrane.org/handbook 